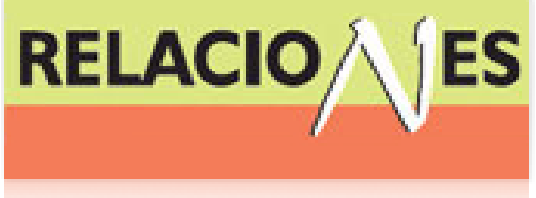

Relaciones. Estudios de historia y sociedad ISSN: 0185-3929

relacion@colmich.edu.mx

El Colegio de Michoacán, A.C

México

Fink, Hady; Boehm, Frédéric

Corrupción en la policía de tránsito. Una primera aproximación a través de entrevistas con taxistas colombianos

Relaciones. Estudios de historia y sociedad, vol. XXXII, núm. 126, 2011, pp. 67-85

El Colegio de Michoacán, A.C

Zamora, México

Disponible en: http://www.redalyc.org/articulo.oa?id=13718501003

- Cómo citar el artículo

- Número completo

- Más información del artículo

- Página de la revista en redalyc.org

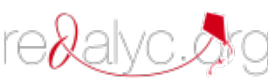

Sistema de Información Científica

Red de Revistas Científicas de América Latina, el Caribe, España y Portugal Proyecto académico sin fines de lucro, desarrollado bajo la iniciativa de acceso abierto 


\title{
Corrupción en la policía de tránsito. Una primera aproximación a través de entrevistas con taxistas colombianos
}

\author{
Hady Fink* \\ UNIVERSIDAD DE PASSAU (ALEMANIA) \\ Frédéric Boehm \\ UNIVERSIDAD DEL NORTE (COLOMBIA)
}

Este texto analiza el fenómeno de la corrupción en la policía de tránsito, a partir del estudio de caso de la interacción entre los taxistas colombianos y los oficiales de tránsito de dos ciudades, Bogotá y Barranquilla. La perspectiva desde la que se aborda el análisis es el del paradigma de la acción racional de la teoría económica. Se utiliza la metodología de "entrevistas a expertos", que permite hacer generalizaciones a partir de los estudios de caso.

(Corrupción, acción racional, entrevista a expertos, taxistas, oficiales de tránsito)

\section{CorrupCión EN LA POLICÍA ${ }^{\mathrm{I}}$}

L

a policía es una institución vulnerable a la corrupción en varios niveles. Esto se refleja claramente, por ejemplo, en la percepción de los ciudadanos alrededor del mundo. Cada año el Barómetro Global de la Corrupción (BGC) de Transparencia Internacional (TI) mide, a través de encuestas, cómo y en qué ámbitos de su vida las personas se ven afectadas por la corrupción.

El Barómetro de 2006, para el que se entrevistaron ciudadanos de América ${ }^{2}$ África, Asia y Europa, muestra de forma contundente que

\footnotetext{
*(Hadyfink@hotmail.com) (fboehm@uninorte.edu.co)

${ }^{1}$ Las opiniones que se expresan en este artículo son responsabilidad exclusiva de los autores y no representan las de las organizaciones a las que están vinculados. Agradecemos a los árbitros anónimos de esta revista por sus valiosos comentarios.

${ }^{2}$ Los países latinoamericanos cubiertos en el 2006 fueron: Argentina, Bolivia, Chile, Colombia, República Dominicana, México, Panamá, Paraguay, Perú y Venezuela. En el 2009 fueron: Argentina, Bolivia, Chile, Colombia, El Salvador, Panamá y Venezuela.
} 
GráficA 1. Porcentaje de personas que informaron haber pagado sobornos, en los 12 meses anteriores, por servicio.

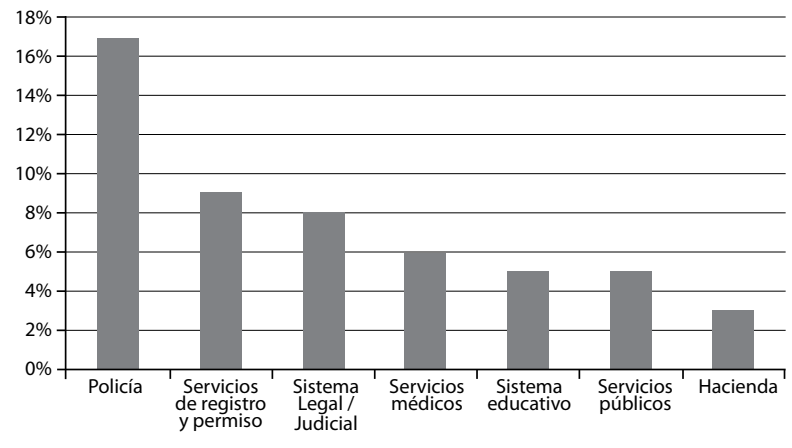

Fuente: Barómetro Global de la Corrupción, 2006.

los sobornos a policías son algo cotidiano, y que son más frecuentes fuera de Europa y Norteamérica. En el mundo, 17 por ciento de los encuestados afirma haber pagado sobornos a policías (gráfica 1), África y Latinoamérica son las regiones más afectadas. En el Barómetro de 2009, el porcentaje global aumentó hasta 21 por ciento.

$\mathrm{El}$ informe de Transparencia Internacional destaca que en Latinoamérica aproximadamente uno de cada tres encuestados que tuvieron contacto con la policía declaró haber pagado sobornos. ${ }^{3} \mathrm{Sin}$ embargo, como lo muestra la gráfica 2, el costo del pago de sobornos a policías en esta región es significativamente menos alto en comparación con lo que se tiene que pagar en otros sectores como los servicios médicos, el sistema legal y hacienda. Pero multiplicado por la frecuencia, queda claro que los sobornos que se pagan a la policía tienen un impacto no despreciable en el bolsillo del ciudadano medio.

Como veremos a continuación, hay varios aspectos y características de las instituciones policiacas que apuntan a que haya un alto riesgo de corrupción. Por supuesto, hay que tener en cuenta por un

${ }^{3}$ Latinoamérica se ubica en segundo lugar entre las regiones. El primero lo ocupa África donde casi 55 por ciento de los encuestados que tuvieron contacto con la policía declaró haber pagado sobornos. 
GrÁfica 2. Latinoamérica. Costo medio del último soborno que se pagó (€).

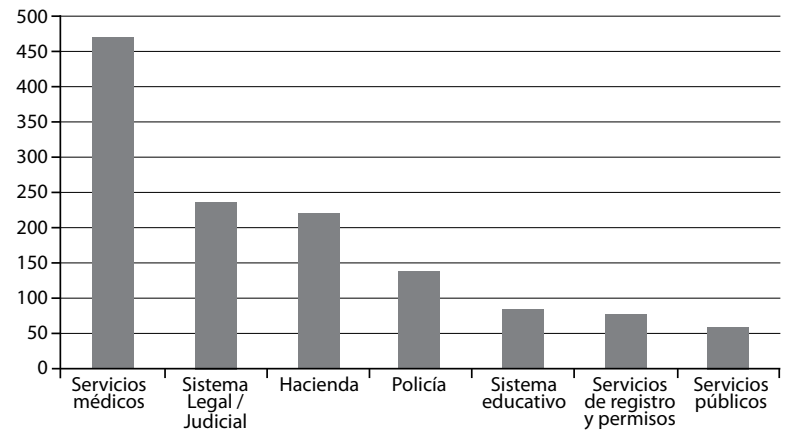

Fuente: Barómetro Global de la Corrupción, 2006.

lado que la corrupción es un fenómeno complejo (véase Boehm y Lambsdorff 2009) y, por otro, que también la policía es una organización compleja con varios niveles y diferentes actores y procesos.

Existe una creciente literatura alrededor del tema. Punch (2004) ofrece una vista general excelente, incluyendo una tipología de posibles actos corruptos en la policía. Quah (2006) muestra los factores que han sido claves para el éxito de las reformas en la policía de Singapur. A menudo, el enfoque se relaciona con el control de delitos del crimen organizado en campos como la prostitución, el tráfico de drogas, el juego, etcétera. En el presente ensayo, el foco se pone sobre un aspecto delimitado pero que es muy visible para los ciudadanos: la pequeńa corrupción ${ }^{4}$ relacionada con la policía de tránsito.

Es importante diferenciar entre el soborno, que es el pago de dinero por un favor corrupto y la extorsión, que es la extracción de sobornos a través del uso de la violencia o de amenazas. Aunque tienen una apariencia similar pues en ambos casos se intercambia dinero entre dos o más personas, los mecanismos que los rigen son muy diferentes. Se paga un soborno para obtener algo a lo cual uno

\footnotetext{
${ }^{4}$ Se le llama también corrupción de pequeña escala, porque cada acción involucra usualmente cifras modestas, aunque el total de su suma puede ser muy alto.
} 
no tiene derecho, o para evitar un costo que se debiera pagar, como puede ser una multa de tránsito. La extorsión, en cambio, se paga para obtener un servicio al que uno tiene derecho -es una especie de impuesto adicional- o como pago por una sanción inventada, que uno no ha cometido.

Recordemos la ecuación simple pero rica en información de Robert Klitgaard: Corrupción $=$ monopolio + discreción - rendición de cuentas (Klitgaard 1988, 116). La policía de tránsito tiene todos esos ingredientes: los policías tienen la autoridad necesaria para abusar de su poder y, por supuesto, no tienen competencia. En caso de una infracción de tránsito los conductores tienen que tratar con el policía que los detuvo, quienes tienen de facto un elevado nivel de discreción.

Es importante diferenciar entre soborno y extorsión porque existen diferencias a la hora de luchar contra cada uno de estos fenómenos. Un conductor que ignora un semáforo puede sobornar a un policía para eludir la sanción oficial. Una posible -y clásica- política anticorrupción, en este caso, consiste en ofrecer recompensas a los policías que sancionen a los conductores que pasan en rojo. Así tienen un incentivo para cumplir con su misión y no dejarse sobornar por "cerrar los ojos". Pero esta política, a priori muy sensata, tiene un efecto perverso: la recompensa incentiva a los policías a detener conductores al azar, acusándolos de una infracción inexistente. La recompensa que debería evitar el soborno lleva a cometer un acto de extorsión. La clave para entender esta transformación del soborno en extorsión es que el conductor no puede demostrar que no se pasó en rojo, porque es la palabra del policía contra la palabra del conductor. Si hubiera cámaras instaladas en cada semáforo habría un control, tanto de los conductores como de los policías, y no se daría esa transformación.

En este ejemplo teórico falla la medida anticorrupción porque no ataca el centro del problema. El cambio implementado no se hace en el sentido de la mencionada ecuación de Robert Klitgaard. En ella el poder monopólico y la discreción son precondiciones necesarias para que la policía de tránsito cumpla con sus obligaciones. Por tanto, en términos de ese modelo, la única opción viable es acre- 
centar la rendición de cuentas, implementando una mayor supervisión de los policías.

En términos de la teoría económica, la corrupción puede entenderse como un crimen calculado (Becker 1968; Klitgaard 1991). Los actores son considerados como "hombres económicos" (homines oeconomici) que tratan de maximizar sus ganancias. Como en cualquier situación, comparan la ganancia esperada de un acto de corrupción con su costo esperado. Si la ganancia supera los costos, preferirán la alternativa corrupta. Por supuesto, es importante anotar que si los individuos realmente siguieran este cálculo costo-beneficio, el mundo sería de hecho muchísimo más corrupto. Sin embargo, partir de este "peor de los casos" es ponerse del lado seguro: no significa que no puedan existir-igracias a Dios!-individuos que por motivos intrínsecos permanecen honestos a pesar de verse enfrentados con una oportunidad corrupta. Sin embargo, contar con esta honestidad es una estrategia arriesgada desde el punto de vista de políticas públicas que quieran mitigar riesgos de corrupción: es una variable no observable a priori, y difícil de influenciar. Sin menospreciar la importancia de los valores y de la motivación intrínseca, los cuales juegan un papel clave a la hora de la decisión de ser corrupto o no, seguimos aquí el modelo del hombre "costo-beneficio". Si se lograría disminuir, a través de incentivos adecuados, el comportamiento corrupto de este "hombre económico" la batalla en gran parte ya estaría ganada. Como lo señalamos en otra parte (Boehm y Lambsdorff 2009, 70): "Existen suficientes razones morales para luchar contra la corrupción, pero la ética por sí sola no proporciona una guía suficiente a los involucrados. Los incentivos deben elevar los riesgos del comportamiento corrupto y resaltar los beneficios de un comportamiento íntegro".

Por cierto, el modelo, en nuestro ejemplo, nos ayuda a explicar la transformación del soborno en la extorsión. Cuando hay una recompensa por cada infracción multada, el razonamiento de los policías es que aceptar sobornos es menos interesante, porque la recompensa ofrecida por ser honestos hace que las ganancias por ser corruptos sean menores, ya que de esas ganancias esperadas hay que deducir la pérdida de la recompensa por ser honestos y multar a los 
conductores. Pero, al mismo tiempo, otro acto de corrupción se convierte en más provechoso: detener a los conductores que no han cometido infracciones. La extorsión es más redituable porque es recompensada.

Esa medida consistente en ofrecer recompensas por cada infracción no sólo puede no ser efectiva para reducir los niveles de corrupción, sino que también puede tener el efecto no deseado de que sean extorsionados conductores inocentes. La lección que hay que aprender de este ejemplo es que la base para implementar medidas anticorrupción efectivas es comprender los cálculos de los actores corruptos.

Ésta es la intención primordial del presente trabajo: explorar la racionalidad de la pequeña corrupción relacionada con la policía de tránsito. Sin embargo, consideramos no menos importante resaltar el instrumento que se usó para este fin: las entrevistas. Así, en un primer lugar presentamos a las entrevistas como un instrumento valioso para la investigación cualitativa del fenómeno de la corrupción; también con la esperanza de incitar más trabajos investigativos similares para enriquecer nuestro conocimiento acerca de la corrupción. Después presentamos y discutimos los resultados de las entrevistas realizadas a taxistas colombianos en las ciudades de Bogotá (en 2005) y Barranquilla (en 2010). Terminamos con la exposición de algunas lecciones aprendidas y el planteamiento de preguntas para futuras investigaciones en este campo.

\section{ENTREVISTAS: MÉTODO PARA UNA PRIMERA APROXIMACIÓN}

El mayor problema relacionado con el estudio de la corrupción es que ésta, en la mayoría de los casos, tiene lugar en la sombra. Además, en los acuerdos corruptos entre dos partes -con excepción de la extorsión-ambas ganan. A priori, no existe ningún incentivo para romper el acuerdo y denunciar el acto de corrupción.

En otras palabras, es difícil obtener datos tanto sobre la incidencia, como sobre los mecanismos que rigen tales acuerdos corruptos. Para tener una idea de la incidencia se pueden usar encuestas de percepción como el Barómetro de Transparencia Internacional, o el Ín- 
dice de Percepciones de Corrupción de la misma organización, el cual se elabora basándose en las percepciones de expertos. Otra manera es contar los casos de corrupción. Pero los estudios que miden casos de corrupción en el sistema judicial realmente no miden el nivel de su incidencia, sino más bien la eficacia de la justicia en procesar casos de corrupción en términos de tiempo medio de duración de los procesos, o el número de casos de corrupción procesados. El problema es que el nivel de corrupción real en un país puede ser más alto que en otro con menos casos procesados. Además, se corre el riesgo de ver únicamente la punta del iceberg, y de tener un sesgo en la muestra: se ven únicamente los casos que por una razón u otra llegaron a la luz del día; ¡los casos "exitosos" no entrarían en esta medición!

Sin embargo, para entender mejor el fenómeno de la corrupción y luchar mejor y de manera más eficiente contra él, es necesario investigar más en detalle las reglas que lo rigen. ¿Cómo hacerlo?

Una opción viable para explorar el fenómeno de la corrupción son los métodos cualitativos de investigación, siempre que se superen los problemas de la neutralidad y la generalización. Una posibilidad son los estudios de caso. Analizar casos de corrupción es una fuente rica en información para encontrar respuestas a preguntas como las siguientes: ¿cómo iniciaron las partes el contacto?, ¿cómo se desarrollaron las negociaciones?, ¿cuáles fueron las razones de que un determinado acuerdo haya fallado? El análisis de acuerdos de corrupción de alto nivel, de su proceso típico en el tiempo y de sus debilidades institucionales en Boehm y Lambsdorff (2009), por ejemplo, se basa en un análisis neoinstitucional de casos de corrupción.

La ventaja general del uso de métodos cualitativos de investigación es que facilitan el acceso a tópicos complejos, clandestinos y tabú (Bogner y Menz 2005a). En las complejas sociedades modernas y postmodernas hay "un creciente número de interacciones humanas que sólo son accesibles a través de métodos cualitativos de investigación" (Atteslander 2006, 48)..$^{5}$ Bajo estas circunstancias los métodos inductivos se muestran más apropiados que los deduc-

\footnotetext{
${ }^{5}$ Todas las traducciones de textos en inglés o alemán son de los autores.
} 
tivos. ${ }^{6}$ Esto es especialmente cierto para tópicos que han sido poco explorados, como los mecanismos de la pequeña corrupción en la policía de tránsito.

Debido a la naturaleza clandestina de la corrupción, el único método cualitativo viable es la recolección de datos verbales. Otros métodos como la observación, o la interacción, no pueden considerarse por la dificultad de llevarlos a cabo. En nuestra investigación usamos un método específico desarrollado por Meuser y Nagler (1991), llamado entrevistas a expertos, el cual exponemos sintéticamente.

Hablando en general, el objetivo de la investigación cualitativa es explorar exhaustivamente un número limitado de casos. Esto tiene la desventaja de que -a diferencia de la investigación cuantitativa- los resultados no permiten formular conclusiones generales que se puedan referir a otros casos que no sean los estudiados. Pero como el objetivo del estudio de la corrupción es la implementación de medidas para combatirla, nosotros pretendemos explícitamente sacar conclusiones acerca del comportamiento, los incentivos y las motivaciones de los policías de tránsito y de sus cómplices. A diferencia de los métodos cualitativos de investigación tradicionales, las entrevistas a expertos permiten la generalización de las conclusiones, dado que su objetivo explícito no es entender un caso particular del sujeto entrevistado, sino más bien obtener "información común supra-individual" (Meuser y Nagel 2005, 80), por medio de la comparación de la información obtenida con otros textos. Para conseguir el objetivo se siguen los siguientes pasos:

a. Transcripción. A diferencia de la generalidad de los estudios verbales cualitativos, la transcripción completa, dadas las circunstancias, puede ser reemplazada por la transcripción de las partes que se considere que son las más importantes.

b. Parafraseo. Las diferentes partes se ordenan en secuencias.

c. Títulos. Se ponen títulos a las secuencias parafraseadas. Con refe-

\footnotetext{
${ }^{6}$ Los métodos inductivos usan la investigación empírica para obtener conclusiones que lleven a desarrollar conceptos teóricos. Los métodos deductivos trabajan al contrario: se obtienen conclusiones de las teorías existentes, que después son probadas empíricamente.
} 
rencia a esta parte, se permite romper la secuencia de la entrevista original, lo cual para otros métodos cualitativos de investigación sería un "pecado mortal" (Meuser y Nagel 2005, 85).

d. Comparación de entrevistas. Secuencias similares de entrevistas diferentes se clasifican y se les da coherencia a los títulos.

e. Conceptualización sociológica. El contenido se formula en términos de generalización empírica, que no está atada a las entrevistas individuales y su terminología.

f. Generalización teórica. Se generan teorías en una última etapa de abstracción.

Según Bogner y Menz (2005b, 36-39), las entrevistas a expertos se pueden usar para diferentes propósitos:

a. Exploración, especialmente de temas nuevos. Este tipo de entrevistas pueden ser muy eficientes para conseguir una comprensión rápida del tema y para hacer hipótesis.

b. Sistematización del tema de investigación. Las entrevistas a expertos proporcionan información adicional sobre el tema que se investiga.

c. Generación de teoría. Los datos obtenidos por medio de las entrevistas a expertos se usan para generar teorías formales.

Además de una implementación conceptualmente correcta, el factor crucial que determina la calidad de los datos obtenidos es la selección de los entrevistados. Este proceso, más bien complejo, no se expone aquí en detalle. ${ }^{7}$ Hablando teóricamente, un experto es alguien "que conoce todo sobre algo, y nada sobre todo lo demás" (Bimazubute 2005, 47). La única medida objetiva para la selección de los expertos es su experiencia relevante para el tema. Muchos años de experiencia pueden considerarse necesarios, pero -como lo han probado algunas investigaciones precedentes- (Lambsdorff y Fink 2006; Fink 2010) de ninguna manera es una condición suficiente para convertirse en experto.

\footnotetext{
${ }^{7}$ Véase Fink (2010) para información detallada sobre el concepto de entrevistas a expertos.
} 
Para el tema que se presenta, que es la corrupción en la policía de tránsito, los conductores de taxis pueden muy bien ser considerados como expertos, porque frecuentemente tienen tratos con los policías.

Después de esta breve presentación de las entrevistas a expertos como método de investigación, reflexionamos ahora sobre nuestras técnicas de recolección de datos empíricos. Como se mencionó arriba, los autores llevaron a cabo entrevistas con un total de 51 taxistas, 25 en el 2005, y 26 en el 2010. Los primeros resultados se evaluaron en el 2005, y se confirmaron con los de la segunda muestra del 2010.

Hay que hacer notar que la selección de los entrevistados (taxistas elegidos al azar) y la atmósfera de la entrevista (viajes en taxis), en un sentido estricto podrían no cumplir los requisitos de la metodología de entrevistas a expertos. Pero como el contenido de las entrevistas no fue muy complejo, su grabación y el análisis de los datos se hicieron conforme a una versión simplificada del método expuesto arriba. Se transcribieron partes y resultados de las entrevistas, se compararon los contenidos y se generalizaron teóricamente.

Los autores son conscientes de que se podrían utilizar objeciones a la metodología para desacreditar los resultados. Pero la notable coincidencia de opiniones entre las dos muestras autoriza las conclusiones que se presentan. Sin embargo, queremos dejar en claro que nuestro trabajo debe entenderse como una primera aproximación, una exploración, para acercarnos a un tema nuevo, y crear hipótesis para nuevas y futuras investigaciones.

Taxistas en Colombia. Primeros resultados basados EN ENTREVISTAS

Colombia no escapa a los resultados mostrados en las mediciones de corrupción por los Barómetros, y las entrevistas con expertos muestran que la corrupción en la policía de tránsito es el sector con mayor incidencia de pequeña corrupción (World Bank 2002).

Los autores de este trabajo optaron por acercarse al problema a través de entrevistas con taxistas. De hecho, las primeras entrevistas fueron un producto adicional de un trabajo de investigación sobre la corrupción en Colombia (Lambsdorff y Fink 2006), y se hicieron 
mientras uno de los autores se movilizaba, en taxi, de una entrevista con un experto -no taxista- a otra. De esta manera se logró un total de 25 entrevistas con taxistas, las cuales se dieron de forma aleatoria y tuvieron lugar en su gran mayoría en Bogotá. Los resultados de estas entrevistas fueron revisados con ocasión de esta publicación. Además, se hicieron 26 entrevistas adicionales y aleatorias en el trascurso de los meses de febrero y marzo del 2010 en la ciudad de Barranquilla, las cuales confirmaron ampliamente los resultados de las primeras. $^{8}$

Las entrevistas abiertas se hicieron alrededor de la posibilidad de pagar un soborno para evitar una sanción después de haber cometido una violación de las leyes de tránsito. También se habló sobre las experiencias vividas en lo que concierne a la extorsión. Los resultados (véase también Lambsdorff y Fink 2006, 22-23) se presentan a continuación.

Primero, sobornar a un policía es un hecho muy común. En la mayoría de los casos es posible pagar un soborno para evitar una multa, y el problema reside más en determinar la cantidad que se tiene que pagar. En otras palabras, los taxistas señalan que cuando son detenidos por un policía de tránsito ya saben que es muy probable que de hecho no sean multados: su preocupación es cuánto le tendrán que pagar al policía como soborno. Este hecho, que se desprende de las entrevistas, parece no haber cambiado mucho en los 5 años que transcurrieron entre las que se realizaron en el 2005, y las llevadas a cabo en el 2010.

Cabe señalar, sin embargo, que en el 2005 los entrevistados en Bogotá mencionaron que la situación cambió después de una reforma: hasta finales de los años noventa el tránsito en Bogotá fue regu-

${ }^{8}$ Estos resultados similares podrían extrañar a conocedores de Colombia. La costa caribe colombiana tiene una cultura muy diferente a la del interior y en particular de Bogotá, y es común escuchar comentarios que apuntan a la Costa como más corrupta que el interior. Sin querer entrar en este debate, sólo queremos resaltar que nuestra investigación no nos dio evidencia de estas supuestas diferencias. Más bien, la similitud en los resultados nos reafirma que el modelo de cálculo costo-beneficio es el camino adecuado: Si los incentivos se prestan a que haya corrupción, esta tendrá lugar sin que el lugar y la cultura local tengan una influencia mayor. De hecho, toda cultura desdeña el abuso que se hace de una posición de poder delegado. 
lado por agentes de tránsito privados. Sus integrantes, a los cuales se referían como "los azules" (por su uniforme) o "chupas" eran considerados mucho más corruptos que los agentes de policía que ahora están encargados de esta tarea. ${ }^{9}$ Lo mismo se aplica al caso de Barranquilla: en vista de la mala imagen de la empresa privada Metrotránsito S.A., y los distintos hechos de corrupción ocurridos durante los ańos 2006 y 2007, ${ }^{10}$ el alcalde de Barranquilla, Alejandro Char Chaljub, tomó la decisión en octubre del 2008 de suscribir un contrato con la Policía Nacional para la regulación y el control del tránsito en Barranquilla. Según información escrita recibida por los autores por parte de la Alcaldía de Barranquilla, la "decisión del arribo de la Policía Nacional para el manejo, control y regulación del tránsito en la ciudad estuvo soportada en las muy buenas experiencias que se han tenido en distintas ciudades del país, entre otros el caso de Bogotá". ${ }^{11}$

Segundo, muchos entrevistados hablaron de lo elevado de las multas en caso de infracción como causa para el intento de eludir una multa a través de sobornos. En comparación con las multas previstas en el 2005, éstas se han incrementado aún más. Las multas, según el Código de Tránsito de Colombia, Articulo 131, están calculadas basándose en el salario mínimo (en el 2005, el salario mínimo fue de 381,500 pesos colombianos, en el 2010, el salario

\footnotetext{
${ }^{9} \mathrm{La}$ ciudad de Cali, que sigue con este modelo de regulación privada del tránsito hasta hoy en día, parece tener problemas similares: "En el 2009 se reportaron 133 quejas, de las cuales 43 eran por extralimitarse en sus funciones, 46 por omitir o retardar asuntos a su cargo y otras 19 (calificadas de gravísimas) por solicitar o recibir dádivas de personas. Según la directora de Control Interno, María Grace Figueroa, estas últimas denuncias corresponden al pequeño porcentaje de los ciudadanos que se atreven a delatar un soborno" (Periódico El País, 14 de febrero del 2010: "Líos que enredan a los guardas de Tránsito").

${ }^{10}$ Por ejemplo, el exgerente de Metrotránsito, Carlos Altamar Arias, fue encontrado culpable de los delitos de interés indebido en celebración de contratos y prevaricato. Según la denuncia que se había formulado contra Altamar, el fraude superaba los $8 \mathrm{mil}$ millones de pesos a través de la adjudicación de plazas para taxis. Altamar fue condenado a 76 meses de prisión, y está recluido desde abril del 2008 en la cárcel de Barranquilla (información obtenida de varios artículos del periódico El Heraldo, Barranquilla).

${ }^{11}$ Es interesante notar, en este punto, que en Barranquilla, la Policía Nacional tomó la decisión de llamar primero a policías del interior de Colombia, y no de Barranquilla o de la Costa. Hasta la fecha de esta publicación siguen con esta estrategia.
} 
mínimo es de 515 mil pesos colombianos). Por ejemplo, conducir un vehículo sin llevar consigo la licencia de conducción, implica una multa de ocho salarios mínimos legales. Quince salarios mínimos es la multa para infracciones como, por ejemplo: estacionar un vehículo en sitios prohibidos; no utilizar el cinturón de seguridad por parte de los ocupantes del vehículo; conducir un vehículo, particular o de servicio público, excediendo la capacidad autorizada en la licencia de tránsito o tarjeta de operación; o conducir un vehículo a velocidad superior a la máxima permitida. Hasta treinta salarios mínimos legales vigentes, o sea 15,450,000 pesos colombianos (más de 6 mil euros), deben ser pagados, por ejemplo, por "guiar un vehículo sin haber obtenido la licencia de conducción correspondiente"; conducir "sin portar los seguros ordenados por la ley"; por no detenerse ante una luz roja o amarilla de semáforo, una seńal de "PARE" o un semáforo intermitente en rojo; por conducir un vehículo sin luces, o el "cambio del recorrido o trazado de la ruta para vehículo de servicio de transporte público de pasajeros, autorizado por el organismo de tránsito correspondiente".

Por supuesto, multas altas no son una excusa para cometer infracciones; sin embargo, se puede adelantar la hipótesis de que, cuando ya se ha cometido una infracción, el incentivo de eludirla a través de sobornos es más grande entre más alta es la multa. Al tomar en cuenta las condiciones económicas de muchos taxistas, ${ }^{12}$ es obvio lo que puede significar para su hogar tener que pagar una multa tan alta.

Tercero, quedó claro en las entrevistas que el riesgo de insinuar interés en una "solución alternativa", es decir corrupta, es casi nulo. El incentivo para intentar sobornar se ve, pues, reforzado tanto por lo cuantioso de las multas (que son a menudo, de facto, impagables), como por las características (discreción y monopolio) que hacen vulnerable el trabajo de un policía por la tentación de la corrupción.

Es interesante observar desde más cerca el mecanismo a través del cual se inicia la negociación entre el policía y el taxista que come-

${ }^{12}$ Por ejemplo, si trabajan un carro ajeno, tienen que pagar una tarifa diaria al dueño del taxi. Según la suerte del día regresan a casa con lo que se necesita para mantener el hogar; ahorros sin ser dueño del carro son casi imposibles. 
tió una infracción. Este proceso fue descrito en varias entrevistas de forma consistente en 2005, y ha sido comprobado en las entrevistas del 2010, a pesar de tratarse de otra ciudad. El primer paso de este ritual consiste en que el taxista empiece a "llorar" sobre la dificultad financiera que tiene y que no va a poder pagar la multa, indicándole así al policía que está interesado en evitarla. En este punto el policía sigue el juego diciendo que a él le toca hacerlo, que esto es su trabajo, sin embargo, aún no empieza a redactar el formato de la multa. Con este simple gesto, que contradice sus palabras, señala su disposición para, eventualmente, encontrar "otra solución". Dependiendo de su habilidad, el taxista encontrará en la mayoría de los casos una manera de establecer una relación personal con el policía. El policía logra entender la "situación difícil" en la cual se encuentra el taxista, tanto como el taxista entiende que el policía tiene que hacer su trabajo.

Tal transformación de la relación formal de un infractor de las normas de tránsito y un policía de tránsito en una relación personal, facilita grandemente un resultado en el que encuentran una manera de "arreglar las cosas". Esto parece ser especialmente cierto porque el resultado no siempre es el pago de un soborno. En muchos casos el policía cambia la infracción por una menor, reduciendo así el monto de la multa que deberá pagar el taxista, sin ninguna ganancia directa para él.

Cuarto, fue interesante encontrar un acuerdo unánime en que las policías mujeres no son, de ninguna manera, más fáciles de sobornar que sus colegas varones -algunos taxistas dijeron incluso que es imposible sobornarlas-. Las explicaciones a esta observación variaron mucho, tanto en Bogotá, como en Barranquilla y son, por tanto, no concluyentes. Algunos taxistas sospechaban que las policías tenían miedo de aceptar sobornos porque sólo recientemente fueron admitidas en el cuerpo, y tenían menos antigüedad en sus puestos. Otras explicaciones fueron que ellas tenían aversión a aceptar sobornos ilegales, o que simplemente tenían un carácter más fuerte o más terco. Uno de los entrevistados en el 2005, dijo que sospechaba que los taxistas no tenían habilidades para hablar con las mujeres.

Por último, las entrevistas en Barranquilla mostraron que el fenómeno de la extorsión es relativamente desconocido, por lo menos 
en el contexto investigado. El abuso del poder para extraer sobornos sin que haya habido una infracción por parte del conductor parece casi inexistente. Por lo menos, de los 26 taxistas entrevistados en Barranquilla ninguno conocía tal situación, o podía decir que conocía a alguien que hubiera tenido este problema. Esto, por supuesto, no significa que no exista el fenómeno de la extorsión en la policía de tránsito en Barranquilla, pero sí indica que es un problema con poca incidencia. Al ser el taxista la verdadera víctima de un policía corrupto, no hay ninguna razón por la cual aquél hable sobre casos de sobornos activos, y se quede callado sobre casos de extorsión, más bien, uno podría esperar un comportamiento contrario: que sería más fácil conseguir información sobre extorsión en entrevistas, que sobre sobornos activos. En algunas entrevistas, sin embargo, los taxistas dijeron que fuera de la ciudad el riesgo de ser extorsionado sí existe.

\section{CONCLUSIÓN Y PRIMERAS LECCIONES}

En el presente trabajo hemos presentado los resultados de entrevistas con taxistas, que se llevaron a cabo en el 2005 (Bogotá) y el 2010 (Barranquilla), acerca de su percepción y sus historias sobre la corrupción en la policía de tránsito. Estamos conscientes de los límites de este trabajo y por ello lo entendemos como una primera aproximación al tema, que requiere una investigación más profunda y detallada. Sin embargo, se pueden extraer algunas lecciones.

Las entrevistas parecen confirmar, en este nivel, un comportamiento racional de los actores involucrados, en el sentido de que toman decisiones basadas en el cálculo racional comparando el valor esperado de su acción con sus costos, tal como lo propone Becker (1968) en su trabajo seminal sobre el crimen desde la perspectiva de la ciencia económica.

Al mismo tiempo, los resultados de las entrevistas parecen demostrar que multas muy altas, por un lado, no tienen el efecto preventivo deseado y, por otro, incitan a la corrupción. Multas caras producen sobornos más altos, y hay poco riesgo, si es que hay alguno, para las dos partes (el control interno de los oficiales de policía no 
es todavía muy eficiente ${ }^{13}$ ). En consecuencia, la utilidad esperada es muy alta para las dos partes, mientras que los costos esperados consisten mayormente en el monto del soborno para el taxista, y son casi nulos para el policía de tránsito. Por lo tanto, con la excepción de algún soborno extremadamente alto pedido por el policía, o alguno extremadamente bajo ofrecido por el taxista, la acción corrupta es la única opción racional para los dos, bajo las condiciones institucionales dadas. Uno podría incluso decir que "sobornar a los policías de tránsito, dadas las circunstancias, no es corrupción sino una consecuencia lógica" (Arteaga, citado en Lambsdorff y Fink 2006, 24).

Esto es especialmente cierto cuando, como en este caso, las partes involucradas son miembros de los estratos sociales de bajos ingresos. En cada incidente, un policía de tránsito puede ganar una semana de salario, mientras que el taxista ahorra también una semana de ingresos pagando un soborno en lugar de la multa (sin hablar de los muy altos montos de las multas mencionadas arriba, que arruinarían a los taxistas y los podrían llevar a tener que vender sus taxis). En consecuencia, no pueden esperarse consideraciones morales de ninguna de las dos partes.

Otra consecuencia implicada en estas observaciones es que el aumento en el monto de las multas no tiene probabilidad de mejorar la situación, porque aumenta los incentivos para elegir la alternativa corrupta dado que sus costos son más bien bajos, por el poco riesgo de ser descubiertos. Parece que en este caso las multas caras no son la mejor medida para prevenir la corrupción. Las multas fijadas en Colombia en este sector son draconianas, por lo que más bien pueden haber aumentado los niveles de corrupción. Más bien, se encontró un acuerdo general entre los taxistas entrevistados en que actualmente es más alto el costo de los sobornos que en ańos pasados. Parece que una de las razones de ese incremento ha sido el aumento en el monto de las multas.

${ }^{13}$ Sin embargo, se creó en la policía colombiana un grupo especial denominado "grupo anticorrupción", integrado por policías, de las diferentes áreas, e investigadores con perfiles en investigación criminal. Su misión es recopilar pruebas de corrupción y luego pasar los casos al sistema judicial. Como incentivo se les paga sobresueldo y se dan garantías para sus familias. 
Las reformas introducidas en Bogotá, y luego en Barranquilla, parecen mostrar mejoras en el manejo general del tránsito en estas ciudades, y en la imagen de los agentes de control. Sin embargo, para el caso específico de los sobornos pagados por haber cometido una infracción de tránsito, los resultados de las entrevistas indican que estas reformas no son suficientes para cambiar, fundamentalmente para ambas partes, el incentivo -creado por las multas muy altas y la probabilidad muy baja de ser descubierto- para buscar y encontrar "soluciones informales". Sería interesante investigar, en el futuro, si una medida como la rotación de personal en las patrullas de policía que controlan el tráfico puede aumentar el riesgo de detección. El hecho de cambiar a los integrantes podría hacer más difícil una colusión entre los policías de una patrulla, y aumentar la probabilidad de que algunos denuncien casos de soborno.

Finalmente, puede ser interesante explorar, también en futuras investigaciones, el tema de las mujeres en la policía como instrumento anticorrupción. Al tomar en cuenta lo señalado arriba, integrar mujeres en las patrullas de policía de forma regular podría cambiar el comportamiento de esas patrullas, por ejemplo, o ayudar a romper redes corruptas dominadas por hombres, reforzando el efecto de introducir una outsider en un sistema establecido. Existe ya una amplia literatura sobre el nexo entre género y corrupción, el cual básicamente gira alrededor de tres preguntas: 1 . ¿Existe alguna diferencia según el género en el comportamiento respecto a la corrupción?; 2 . ¿impide la corrupción una equidad entre los géneros y de qué forma?; y 3 . ¿existen diferencias según el género en el impacto de la corrupción? Los resultados de las entrevistas del presente trabajo parecen indicar una diferencia entre hombres y mujeres en lo que concierne a la pregunta número uno. Otras diferencias en el comportamiento ya han sido observadas en experimentos económicos, de las cuales se podría sacar algún provecho (Frank, Lambsdorff y Boehm 2010).

Queda la pregunta acerca de en qué medida estas conclusiones se pueden generalizar. Ciertamente, el caso analizado no es representativo del sector público colombiano entero, porque la policía de tránsito es un caso muy específico y el entorno institucional general no es tan malo (poder monopólico del empleado público y bajo costo, 
de facto, de las acciones corruptas debido a la poca probabilidad de ser descubiertas).

Sin embargo, la posibilidad práctica de transformar una relación formal en una privada, puede ser, hasta cierto punto, generalizada. Como no parece depender de las situaciones específicas de los actores, ni de diferencias culturales -las cuales definitivamente existen entre Bogotá y Barranquilla- se puede esperar que este tipo de comportamiento también facilite la corrupción en otras áreas de la sociedad colombiana, en las que el contacto personal es necesario en una transacción oficial (Lambsdorff y Fink 2005). Este fenómeno problematiza los sectores públicos también en otros países sudamericanos.

Aunque puede no ser únicamente colombiano, y puede no aplicarse a todas las partes del sector público de la misma manera, ciertamente parece ser parte del meollo del problema de la pequeña corrupción en Colombia.

\section{REFERENCIAS}

Atteslander, Peter, Methoden der empirischen Sozialforschung, 11 edición, Berlín, Erich Schmidt Verlag, 2006.

Becker, Gary, "Crime and Punishment: An Economic Approach", The Journal of Political Economy 76 (2), 1968, 169-217.

Bimazubute, Raymond, Die Nachbereitung von Experteninterviews im expertenzentrierten Wissensmanagement, Universidad de Erlangen-Nürnberg, 2005.

Boenm, Frédéric y Johann LambsdorfF, "Corrupción y Anticorrupción: Una Perspectiva Neoinstitucional”, Revista de Economia Institucional 11 (21), 2009, 45-72.

Bogner, Alexander y Wolfgang Menz, "Expertenwissen und Forschungspraxis: die modernisierungstheoretische und methodische Debatte um die Experten. Zur Einführung in ein unübersichtliches Problemfeld" en Alexander Bogner, Beate Littig, Wolfgang Menz, eds., Das Experteninterview. Theorie, Methode, Anwendung, 2a. edición, VS Verlag Wiesbaden, 2005a, 7-29.

, "Das theoriegenerierende Experteninterview. Erkenntnisin- 
teresse, Wissensformen, Interaktion", en Alexander Bogner, Beate Littig, Wolfgang Menz, eds., Das Experteninterview. Theorie, Methode, Anwendung, 2a. edición, VS Verlag Wiesbaden, 2005b, 33-70.

Fink, Hady, "Baby Blues", en Brasilien: Eine empirische Untersuchung sozialer und kultureller Einflussfaktoren, Universidad de Passau, 2011.

Frank, Björn, Johann Lambsdorfa y Frédéric Boehm, "Gender and Corruption-Lessons from laboratory corruption experiments", European Journal of Development Research, publicado en internet el 30 de septiembre de 2010.

Kuitgaard, Robert, "Adjusting to Reality: Beyond 'State Versus Market”, en Economic Development, San Francisco, 1991.

Klitgaard, Robert, Controlling Corruption, Berkeley, Los Ángeles, University of California Press, 1988.

LambsdorfF, Johann y Hady Fink, Combating Corruption in Colombia: Perceptions and Achievements, Discussion Paper, núm. V-44-06, Facultad de Economía, Universidad de Passau, 2005.

Meuser, Michael y Ulrike Nagel, "ExpertInneninterviews-vielfach erprobt, wenig bedacht", en Alexander Bogner, Beate Littig, Wolfgang Menz, eds., Das Experteninterview. Theorie, Methode, Anwendung, 2a. edición, VS Verlag Wiesbaden, 2005, 71-93 (reimpresión, primera publicación 1991).

Punch, Maurice, "Police Corruption and its prevention", European Journal on Criminal Policy and Research 8, 2000, 301-324.

QuAH, Jon, "Preventing Police Corruption in Singapore: The Role of Recruitment, Training and Socialisation", The Asia Pacific Journal of Public Administration 28(1), 2006, 59-75.

World Bank, Colombia: The Economic Foundation of Peace, Washington, The World Bank, 2002.

FeCha de ReCEPCión del aRTíCulo: 30 de junio de 2010

FECHA DE ACEPTACIÓN Y RECEPCIÓN DE LA VERSIÓN FINAL: 24 de enero de 2011 